\title{
Delimitando al concepto de Alfabetización: Una propuesta para un mejor entendimiento
}

\section{Delimiting the concept of Literacy: A proposal for a better understanding}

José Luis Aguilar Trejo ${ }^{\text {a }}$

https://orcid.org/0000-0003-3210-064X

\begin{abstract}
Abraham Moctezuma Franco ${ }^{\text {b }}$
https://orcid.org/0000-0002-4398-4340
\end{abstract}

Benemérita Universidad Autónoma de Puebla, México.

a jose.aguilart@,alumno.buap.mx

babrahammoctezuma@yahoo.com.mx
Recibido: 03/06/2020

Aceptado: 19/09/2020

\section{Resumen}

Lo "informacional" no es el equivalente de "alfabetización". El siguiente artículo analiza al concepto de "alfabetización" desde la desarticulación de lo que se ha venido nombrando como "alfabetización informacional". Creemos que es importante la comprensión y definición de la "alfabetización" porque vivimos en una época en la que su uso y aplicación se ha distorsionando al grado de hacerla contradictoria con su verdadero sentido, una consecuencia de esto, es el manejo incorrecto que se hace de ella al asemejarla con lo "informacional". Por lo tanto, a lo largo de todo el documento se desarrollará y se expondrá qué es y cómo debe entenderse a la "alfabetización". El objetivo es concientizar a la comunidad académica sobre una propuesta conceptual que puede ayudar a un estudio más claro y organizado sobre este fenómeno, el cual tiene raíz en el área educativa, pero con presencia en las demás disciplinas académicas, así como en los diferentes ambientes y contextos en el campo del conocimiento.

Palabras clave: Alfabetización, TIC, multialfabetización, ambiente impreso, ambiente digital.

\begin{abstract}
The "informational" is not the equivalent of "literacy". The following article analyzes the concept of "literacy" from the disarticulation of what has been called "information literacy". We believe that the understanding and definition of "literacy" is important because we live in an era in which its use and application has been distorted to the degree of making it contradictory to its true meaning, a consequence of this, is the incorrect handling that is done hers by likening her to the "informational." Therefore, throughout the document, what "literacy" is and how it should be understood will be developed and exposed. The objective is to make the academic community aware of a conceptual proposal that can help a clearer and more organized study of this phenomenon, which is rooted in the educational area but with a presence in other academic disciplines, as well as in different settings and contexts in the field of knowledge.
\end{abstract}

Key words: Literacy, ICT, multi-literacy, print environment, digital environment. 


\section{Introducción}

Dentro del área educativa, y otras disciplinas, se logra identificar la existencia de una Alfabetización digital, alfabetización en TIC, alfabetización virtual, alfabetización computacional, alfabetización informática y demás compuestos, estos sólo son algunos ejemplos de cómo el término "alfabetización" ha sido manipulado deliberadamente. En los conceptos citados, se puede mencionar que cada uno cuenta con un significado inestable que tiende a difuminarse entre los demás, ya que las diferencias o similitudes se establecen de acuerdo a la visión de cada autor, pero sin considerar un control o unificación de lo que se está exponiendo. Es decir, tropezamos con una variedad de definiciones que motivadas por el deseo de "innovar" se postulan como nuevas o transformadoras, no obstante, el único cambio que tienen es en el nombre, puesto que dejan de lado la importancia de construir ontológicamente un objeto, en este caso, el de la "alfabetización".

De este modo, se logra visualizar una problemática que debe atenderse antes de continuar con el diseño de más proyectos que involucren la enseñanza y aprendizaje de la lectoescritura. Mientras no se delimite adecuadamente lo qué es y cómo debe entenderse la "alfabetización" no se podrá avanzar más en este tema, antes bien, se quedará atascado en una red cada vez más confusa y densa.

Entonces ¿qué es la alfabetización? Esta pequeña pregunta detona el análisis que se ha construido sobre este concepto, además ayudará a entender de mejor forma el objetivo de este artículo. Para responderla invocamos la definición de López y Pérez (2014), quienes explican el nivel más elemental de la "alfabetización", ellos consideran que es el proceso que implica que un sujeto adquiera los conocimientos necesarios de la lectoescritura para desempeñar una comunicación efectiva con la comunidad en la que se encuentra. En este punto hay que ser analíticos, ya que si bien esta definición por un lado es limitada, por otro llega a ser objetiva dado que especifica la raíz de este término. Esto es algo que no debe perderse de vista para, más adelante, definir y delimitar a la "alfabetización". Cabe aclarar que la delimitación para nada equivale a un ejercicio que minimice o "limite" al conocimiento, más bien, es una tarea que conlleva un pensamiento más ordenado y claro. Muy similar a la analogía de limpiar y aplanar un camino que se ha llenado de maleza y baches, en el comparativo el camino es un pensamiento que al ser despejado facilita el trayecto hacia una reflexión que genere conocimientos objetivos y resuelva eficientemente problemáticas sociales y/o educativas.

Ahora, si continuamos con la dinámica de articular el significado de la "alfabetización", podemos decir que, con Ferreiro (1997), escalamos a un nivel más complementario del proceso de lectoescritura. Emilia Ferreiro aporta que el estar alfabetizado no es únicamente aplicar la lectura y la escritura para ejecutar una simple comunicación lineal, sino que esto debe incorporar más elementos que doten a la persona de la habilidad y capacidad para interactuar más con la información que la rodea. Se debe agregar que la finalidad es que el sujeto se apropie significativamente del conocimiento y de los códigos específicos que, en conjunto, le permitan desenvolverse productivamente en su entorno.

Con esta explicación comenzamos a distinguir la manera en que la "alfabetización" pasa de ser un proceso básico a uno más amplio y complejo, pero es precisamente esta transición conceptual la que se postula como el parteaguas de lo que hoy en día se concibe como el conocimiento turbio y enmarañado de la "alfabetización". La propuesta de Ferreiro (1997) es acertada por la profundidad que da al concepto de "alfabetización", sin embargo, también es el punto de partida y la base de varias definiciones - mal dirigidas - de la "alfabetización" (lo que en este documento se entiende como "compuestos incorrectos") ya que más que aportar al estudio y entendimiento de la misma, solo genera confusión.

\section{Método}

El presente documento académico se planeó a partir de una revisión documental extensa que abarcó la exploración de específicamente artículos científicos arbitrados, libros y tesis sobre el tema de alfabetización, estos, dentro de un margen temporal de diez años (2009 a 2019). La búsqueda de estos documentos se realizó desde la base de datos de EBSCO, apoyándose del motor de búsqueda de Google Académico.

Las palabras clave para optimizar aún más la búsqueda fueron: Alfabetización, alfabetización informacional, alfabetizacióndigital, alfabetización educativa y literacidad; a partir de estas palabras 
se identificaron diferentes documentos que fueron seleccionados de acuerdo a su pertinencia discursiva identificada a través de una primera lectura. Una vez efectuada la discriminación de los artículos, se efectuó una serie de lecturas rápidas, a profundidad y de repaso desde dos núcleos temáticos importantes, el ambiente impreso y el ambiente digital.

Desde estas variables se construyó el análisis y reflexión sobre el concepto de alfabetización, lo cual impactó en los resultados, identificando que desde estos dos ambientes, al alfabetización se irá articulando con sus respectivos procesos y cualidades, ideas que se explicarán a continuación.

\section{Desarrollo}

Para iniciar este análisis se plantea la siguiente pregunta ¿cómo se ha llegado a diversificar e intervenir tanto un concepto que se trabajó desde antes de la década de los años 50's? La respuesta lógica a esto sería por la evolución de la sociedad y de los contextos a partir del paso del tiempo, enfoque que ha sido plasmado en la basta literatura académica y que se ha expuesto perfectamente a través de los siguientes autores. En primer lugar se puede rescatar a David Bawden en su documento "Revisión de los conceptos de alfabetización informacional $y$ alfabetización digital", él menciona que el término de alfabetización se puede ubicar en tres niveles, uno donde solo se necesita la capacidad de leer y escribir, otro donde es necesario el uso del código para aplicarlo a la par de otras habilidades o competencias, y un último, que se ancla en la capacidad de aprovechar la alfabetización para generar aprendizaje significativo (Bawden, 2002). Sin duda, esta propuesta sirve como pauta para entender las demás revisiones que se han hecho de este concepto de alfabetización.

En este sentido, Braslavsky (2003) con su documento "Lectura y vida", refuerza el discurso de David Bawden y genera un análisis sobre la evolución de la alfabetización, identificando que esta ha ido cambiando a conforme los contextos sociales se han ido modificando, dando paso a conceptos regidos por el código, lo laboral, lo capitalista y/o industrial, y por último lo contextual; del mismo modo, Carrasco y López (2014) en "Leguaje y Educación: Temas de investigación educativa en México", hacen una extensa revisión sobre cómo la alfabetización se ha ido trabajando, diferenciándola del alfabetismo y de lo que hoy en día se conoce como literacidad, de este modo, se comprende que la alfabetización se ha complementado y ampliado conceptualmente a lo largo de la historia, plasmando en sus definiciones lo que en un entonces es necesario socialmente. Otro aporte interesante de Carrasco y López (2014), es el de hacer énfasis en el significado de la literacidad, propuesta que debe ser cuidadosamente analizada para no caer en errores de uso y aplicación, puesto que es común igualar a la alfabetización con la literacidad.

Sobre la misma línea de revisiones conceptuales, Del Pilar y Pirela (2016) en "La alfabetización como estrategia de inclusión socio-digital: entre diversidades y mutaciones conceptuales", se hace énfasis en cómo la alfabetización recibe un "apellido" a partir de la específica actividad en la que se ejecute, por ejemplo, al relacionarse con los medios masivos de comunicación, con las fuentes digitales de información, o bien, con la manipulación de las TIC emergentes; de esta forma, se dibuja con más fuerza que la alfabetización se va nutriendo de lo que contextualmente y socialmente se va exigiendo para contar con una incorporación e interacción efectiva en determinada etapa histórica.

Por último, se localiza a Reis, Pessoa y Gallego (2019) con "Alfabetización y competencia digital en Educación Superior: Una revisión sistemática", quienes exploran cómo se ha estudiado el concepto de alfabetización desde la era totalmente impresa, hasta la presente etapa digital, concluyendo que, a pesar de la basta investigación que se hace sobre este tema, aun no existe una definición "sustantiva" sobre la alfabetización, entendiéndose como la falta de una delimitación correcta de este término.

Pero habría que ser más críticos y reflexivos con este fenómeno que, más que soluciones, solo ha provocado otro tipo de retos y problemas. A pesar de tantos años de estudio y apoyo por organismos internacionales, nacionales y locales, aún no se ha logrado consolidar una definición de la "alfabetización" que verdaderamente promueva la lectura y la escritura del entorno como el puente para crecer personal y/o profesionalmente.

En este sentido, gran parte de este estancamiento se debe a un descontrol terminológico que se originó justamente cuando la "alfabetización" se comenzó a entender como: el conjunto de actitudes, aptitudes, 
conocimientos, habilidades y demás elementos para desempeñar, a través del acceso y uso de códigos particulares, una labor especializada. Por ejemplo, en el contexto musical se propuso a la alfabetización musical como aquella que desarrolla el aprendizaje necesario en las personas para que transiten sin complicación con todo lo que implica saber cómo leer una partitura, o cómo identificar ciertas palabras y populismos que se utilizan en ese contexto especial; o bien, también se puede ejemplificar a la alfabetización audiovisual, donde se busca formar a los sujetos para que sepan lo que implica capturar, editar y compartir un producto visual. De esta forma, conforme ciertas dinámicas evolucionaban y se colocaban como emergentes, en reacción surgía una "nueva" "alfabetización" para cubrir las demandas cognitivas y técnicas que exigían esas tareas especializadas, por lo tanto, esto originó que se disparara la propuesta de un gran número de alfabetizaciones en distintas disciplinas y campos sociales, lo cual saturó la naturaleza del concepto único de la "alfabetización". Tanta definición colocó a este concepto como un ente amorfo y opaco.

Sobre esta misma línea de propuestas conceptuales que aportaron al nudo ontológico de la "alfabetización", también se empieza a hablar de una alfabetización informacional. De acuerdo a Molina y Valdés (2010) ésta respondió a la necesidad de preparar a los estudiantes en el uso y manipulación de la información, hecho que se convirtió en primordial en el contexto universitario. Este concepto parte de que un desempeño profesional correcto y productivo depende de cómo se busca, selecciona, lee, discrimina, escribe y comparte toda la información que existe, la cual, actualmente se presenta en distintos formatos y ambientes. Es precisamente esta definición compuesta la que ayudará a entender qué es y cómo debe entenderse la "alfabetización", ya que al desarticular a la "alfabetización informacional" se analizaran los elementos que la componen, lo que dará paso a una mejor comprensión de las partes que corresponden a la "alfabetización" y las que pertenecen a lo "informacional".

\section{Resultados}

En este sentido, desde los resultados a partir de la revisión de la literatura, uno de los primeros elementos a identificar es el ambiente, el cual se ancla como un factor con gran impacto e influencia en la "alfabetización".

\section{El ambiente}

A partir de la idea anterior - que refiere a la importancia de distinguir la información a través de sus distintos formatos y ambientes - se encuentra el momento oportuno para profundizar en estos aspectos informacionales que han marcado una época en la que los datos ya no están únicamente en forma física (como revistas, antologías o libros impresos) sino que la difusión del conocimiento y otro tipo de contenido se expandió aún más con las Tecnologías de Información y Comunicación (TIC) digitales. Por lo tanto, la visión de la alfabetización crece, pero hay que aclarar que no cambia, puesto que el sentido de este concepto siempre ha sido y debe ser el mismo independiente del ambiente en el que se aplique, punto que se tratará más adelante.

Antes de continuar avanzando, hay que especificar que entendemos como ambiente a las condiciones particulares que configuran de cierta manera a los procesos de lectura y escritura, por lo que a partir del ambiente (físico o virtual) es como la "alfabetización" va a tomar cierto sentido, pero no significado. Para hacer más entendible esto, hay que pensar a la "alfabetización" como a la agronomía: gran parte de la aplicación de esta ciencia requiere de conocer las características ambientales de distintas zonas geográficas, pues de esto depende el cómo se configurará la práctica agrónoma para ejercer y aprovechar eficaz y eficientemente la humedad, el PH o la densidad de la tierra, por ejemplo. De este modo, la agronomía no se está re-conceptualizando por el medio, sino que solo se está configurando, esto mismo pasa con la "alfabetización". El medio configura, no conceptualiza.

Lau y Cortés (2009) aciertan en exponer que la "alfabetización informacional" llegó a tener importancia en un ambiente impreso que necesitó de una educación que enseñara a los estudiantes a buscar un ejemplar físico dentro de las bibliotecas. Efectivamente, la biblioteconomía se posicionó como aquella ciencia que facilitaba a las personas los conocimientos para desempeñar activa y productivamente el proceso de ingresar a la biblioteca, localizar los catálogos y saberlos manipular, identificar los códigos de ubicación, buscar el piso o los pasillos con sus respectivos estantes, hallar el estante correcto y finalmente encontrar el libro para empezar a darle lectura. Todo este camino representó solamente el proceso 
de búsqueda de la información en el ambiente impreso. De ahí seguía el de lectura rápida para evaluar si la información era acorde a lo que se estaba buscando, lo cual necesitaba de ojear de forma rápida el libro, consultar el índice, o bien, hasta dar una lectura rápida al prólogo.

Aquí se comienza a dibujar la idea de que la "alfabetización" se compone de diferentes procesos. Estos distintos tratamientos se verán estructurados dependiendo del ambiente en el que se establezcan, justo como se acaba de comprobar al describir los procedimientos de la "alfabetización informacional" como el de búsqueda y lectura rápida que se enmarcan por las condiciones del ambiente impreso de la biblioteca física. Ahora, si esta misma dinámica de buscar información en una biblioteca se traslada al ambiente virtual, se encontrará que los procesos serán otros o habrá unos que destaquen sobre los demás, si estos se comparan con los del ambiente impreso.

\section{Procesos en el ambiente impreso}

Enfocando la mira hacia el ambiente impreso, se propone determinar que los procesos más significativos en esta dinámica son los de lectura analítica y lectura crítica. Esto se sustenta a partir de establecer a la "alfabetización informacional" como aquella que busca crear personas que sepan gestionar la información (Martín, 2018), lo cual es una idea similar al campo que trabaja la biblioteconomía. El factor anterior resulta coherente, puesto que, sin importar el ambiente, siempre el proceso de búsqueda será indispensable para tener contacto con la información deseada. Cabe señalar que, si bien la búsqueda de la información es indispensable, este proceso se torna más complejo y esencial en el ambiente virtual, hecho que se analizará en párrafos posteriores.

En este sentido, la diferencia del proceso de búsqueda entre ambientes marca la guía para ir más a fondo, y lograr así responder por qué destacan más en este ambiente impreso los procesos de lectura analítica y crítica. Y es que en un ambiente donde los documentos cuentan con un espacio físico como la biblioteca escolar o local - la búsqueda de estos materiales es laboriosa pero sencilla, ya que se tiene la certeza de que al menos existe algún filtro de confiabilidad de los datos impresos, por lo que se genera un tipo de garantía de información sustentada y/o veraz. Ahora, es aquí donde se inserta el valor de la lectura analítica y crítica, ya que un proceso de acceso a la información siempre tendrá el riesgo de caer en el consumo de contenido no confiable o veraz (Aguaded y Romero, 2015).

¿Pero por qué recuperar lo analítico y lo crítico en documentos que tienen un filtro formal o académico? La respuesta remite a un punto que se ha ido arrastrando a lo largo del tiempo y que aún mantiene presencia en las dinámicas educativas o cotidianas, por lo que su análisis se considera pertinente. Este, refiere a que se la ha brindado una confiabilidad peligrosa a los libros o demás documentos impresos, de modo que una pasta y una portada resultan ser, para la mayoría, los únicos sellos que convierten al contenido de esas hojas impresas en verídico, de esta manera, se descartan los ejercicios analíticos y críticos de la lectura, provocando que el consumo sea ciego y pasivo.

No se descarta que en una época donde la información no se masificaba, cuando aún era exclusiva, esta podía ser consumida con cierta confiabilidad, pero incluso así, en ese espacio temporal, se leía, se analizaba, se criticaba, se cuestionaba y se replanteaba el discurso, no obstante, pareciera que esta dinámica se ha dejado de realizar, olvidando que la producción de la información cuenta con una variable humana que puede cometer errores intencionales, o no. Es así que se identifica una tendencia contradictoria, ya que se puede visualizar cómo a menor información mayor era el análisis y la crítica, pero, actualmente, a mayor información menor es este ejercicio de lectura profunda. Por tal motivo, la proliferación de materiales impresos genera un nuevo reto en los estudiantes de cada uno de los niveles educativos. Tomando en cuenta esta tendencia, el paso del tiempo demandará cada vez más una lectura que evalúe si ese libro impreso contiene datos confiables, tal como lo mencionan Sánchez, Sánchez, Méndez y Puerta (2013), la clave para evaluar, clasificar, argumentar, cuestionar y aplicar la información en contextos específicos, es la lectura crítica.

Por lo tanto, no es adecuado que se continúe con este tipo de prácticas que de forma automática validan todo lo que está sobre hojas de papel y cubiertos por una pasta con diseño editorial. Hay que ser analíticos y críticos y más en un ambiente impreso que se está descuidando al grado de encontrar con mayor frecuencia libros de texto oficiales con errores o información incorrecta, antologías con 
datos desactualizados, ejemplares con discursos dudosos, etc. No hay que perder el enfoque y ser más curiosos con lo que nos rodea para hacer de la lectura crítica y analítica un proceso orgánico. Sobre esta idea versa la siguiente cita:

Me gustaría que a partir de ahora dudaras de todo. Que leyeras lo que diré de arriba abajo, con escepticismo, resistencia, escudriñando cada dato. No se trata de creerse con fe todo lo que dice ningún libro, ni de repetirlo después como una canción. Importa más desarrollar una opinión propia, original, matizada, fundamentada, diferente. Quizá imperfecta o incompleta, pero coherente con nuestra experiencia personal. (Cassany, 2006, p. 19)

Es claro que educativamente se debe hacer frente a la actitud negativa de consumir información sin ser analíticos y críticos, ya que de esto depende el crecimiento de un conocimiento que verdaderamente aporte a lo social.

Es así que, a partir de la desarticulación de la "alfabetización informacional" ya se pueden ir organizando, poco a poco, los elementos que configuran al concepto de la "alfabetización", los cuales hasta el momento son el ambiente y los procesos. Es decir, que parte de los resultados de la revisión de literatura, arrojan que la "alfabetización" se va perfilando como un concepto que se construye de algunas variables, por lo que hay que poner atención a estas para identificar con qué se está lidiando. De este modo, se ha visto que la "alfabetización informacional", en un ambiente impreso, va a implicar distintos procesos como el de búsqueda, selección y producción de la información, pero postula como esenciales a los procesos de lectura analítica y crítica por las condiciones que alberga este entorno físico. Pero hace falta agregar dos elementos más para complementar la configuración de la "alfabetización": las cualidades y el contexto.

\section{Cualidades y el contexto en el ambiente impreso}

Estos elementos llegan a aportar cierta dirección o particularidad a la "alfabetización", pues dependiendo el contexto se necesitarán de ciertas cualidades con las que las personas deben contar para desenvolverse efectivamente en el círculo social $y / 0$ en actividades específicas. Cabe diferenciar que el término de contexto no debe confundirse con el de ambiente, puesto que el contexto abarca aspectos sociales y culturales, los cuales pueden modificarse por el ambiente que, como ya se explicó, engloba más el entorno o espacio.

Por ejemplo, con base a la revisión bibliográfica que se realizó para desarrollar un estado del arte de la "alfabetización" en la educación superior, en primer plano, se puede definir que el contexto a priori será el de la educación superior, en el cual podrán coexistir contextos más específicos que surjan a partir de las áreas, disciplinas, materias, cursos, grupos, círculos, etc. Ahora, esta diversidad de macros y micros contextos, van a ser configurados y se van a diferenciar si se exploran en ambientes diferentes, en este caso el impreso y el virtual, ya que las prácticas sociales van a configurarse de otro modo dependiendo en dónde se apliquen.

Una vez entendiendo cómo intervienen los elementos del contexto y del ambiente, se puede exponer -y al mismo tiempo ejemplificarque dentro del contexto de la educación superior existirán ciertas cualidades para desempeñar actividades informacionales, que en el ambiente impreso - a diferencia del virtual — se dibujan las de socialización, colaboración y liderazgo. Esta idea se fundamenta conjugando el discurso de autores como Carlino (2013, 2017), quien afirma que en cada disciplina el estudiante necesita de ciertas prácticas letradas para entender y participar activamente con sus pares y con los profesores, ya que sin este tipo de conocimientos especializados, el transitar del estudiante por la carrera puede tornarse complicado. $Y$ es que la lectura y la escritura no debe limitarse al lápiz o a la hoja, o bien al teclado y la pantalla, sino que más bien el leer y escribir debe representar una tarea que puede aplicarse en todo, puesto que todo lo que nos rodea es información, por lo tanto, todo se puede leer y codificar. Por ello, resulta coherente pensar que: 1) un estudiante de cualquier disciplina, debe saber leer y analizar los textos especializados como lo apunta Olave, Cisneros y Rojas (2013, p.460).

En la universidad el estudiante se enfrenta a amplios volúmenes de información que, si bien están delimitados por el docente con relación a la sugerencia de los materiales de lectura, estos pertenecen a un extenso corpus de conocimiento del que se debe tener cierto dominio, en ellos cobra importancia el autor y la época en que fue escrito, además del diálogo 
que se establece con otros autores y otros saberes; son textos que no han sido escritos para el estudiante sino para la comunidad académica y que requieren habilidades de clasificación, comparación, esquematización y análisis, entre otros, para ser comprendidos.

Y 2) leer las dinámicas entre académicos, las tendencias políticas dentro de la institución, los códigos, las tradiciones, etc., para acceder sin problema a la otra información de la disciplina que sobrepasa a la letra.

Entonces, el contexto se convierte en un mediador que condiciona a la alfabetización para que esta se ajuste a modo de ser la herramienta ideal para leer y escribir toda la información que se alberga en ese espacio especial. Por otra parte, en el caso de las cualidades que el contexto de la educación superior en un ambiente impreso necesita, se encuentra a la socialización, colaboración y liderazgo como las principales, ya que Fernández y Piña (2014) refieren que el acceso, producción y difusión de la información necesita de la interacción y colaboración para el intercambio de ideas y propuestas, dentro o fuera de la academia, como lo señalan Vega y Quijano (2010) y Osuna, Frau y Marta (2018). Por el lado del liderazgo, se concuerda con Ponjuán y Vera (2012), quienes colocan a esta cualidad como eje para que la socialización y la colaboración marchen sin complicaciones, puesto que un buen líder sabrá cómo mediar y delegar las tareas o responsabilidades dentro del grupo para aprovechar al máximo las capacidades de todos los integrantes.

\section{El ambiente virtual y sus procesos.}

Haciendo una breve recapitulación, se encuentra que la "alfabetización" no se va a limitar a la lectura y escritura de la letra, sino que va más allá, plasmándose como el fenómeno que engloba la identificación, manipulación y aplicación de los códigos que componen cada uno de los aspectos de este mundo, puesto que todo es información, y todo se puede codificar. De este modo, se fue mostrando — con la desarticulación de la "alfabetización informacional en lo impreso-que la "alfabetización" se puede posicionar en distintos ambientes y contextos, los cuales, configurarán al concepto de la "alfabetización" de manera que éste estructurará procesos y cualidades específicas para que la persona se desenvuelva efectivamente. Para reforzar aún más esta idea, se hace el mismo ejercicio, pero ahora en el ambiente virtual.
Hay que recordar que en el ambiente impreso, el proceso de búsqueda se postuló como básico, laborioso pero no complejo, esto cambia en el ambiente virtual, ya que el proceso de búsqueda se convierte en esencial en un entorno donde la información se multiplica a una velocidad impresionante $y$ se diversifica en diferentes formatos, los cuales se albergan en distintos sitios. En este sentido, Pini (2019) acierta en decir que solo basta con contar con un dispositivo digital que permita el acceso a la información virtual, y con ello, adentrarse a un mundo extenso de datos por leer y analizar. Pero es esta misma profundidad del espacio virtual la que resulta un reto para el proceso de búsqueda, el cual debe ser cuidadoso y apoyado por una lectura crítica que promueva la selección de los documentos veraces de los no apropiados. A manera de ejemplo, Cassany (2006) asemeja esta labor con la búsqueda de joyas debajo del fango que está en las profundidades del mar, puesto que no todo lo que existe en Internet es correcto, confiable y productivo, por lo que en el proceso de la navegación uno se puede encontrar con materiales con información que no aporten al desarrollo personal y profesional de un estudiante (López y Parker, 2009).

Y es que no se está diciendo que el consumo de la información incorrecta $-o$ la que tiene simplemente finalidades de entretenimiento per se - sea un acto negativo, más bien lo que hace contraproducente a esta información es la facilidad con la que las personas pueden engancharse con su contenido. Es lo que Igarza (2009) nombra como las "burbujas de ocio" las cuales abarcan gran parte del tiempo productivo de cualquier persona. Estas "burbujas", incluso, llegan al grado de desviar la búsqueda y dificultar el hallazgo de algún documento verdaderamente confiable y productivo para la finalidad formativa o educativa de un estudiante.

Es así que la búsqueda dentro de una labor informacional en lo virtual será un proceso importante a desarrollar, más que en el impreso. Ahora, conforme a Hernández, Cassany y López (2018) es necesario contar con los procesos desarrollados de lectura crítica para hacer frente al mar de información en Internet y seleccionar la información más útil y confiable; lo que Scalerandi (2018) entiende como la capacidad de validar los contenidos a través de los procesos de lectura crítica y selección. 
Por último, un proceso más a destacar que se inserta en lo informacional dentro de un ambiente virtual, es el de producción. Siguiendo la lógica de Gómez y Gómez (2015), los medios de comunicación digitales requieren cada vez más de específicas formas de escritura e interacción, por tanto, la producción de la información se torna más amplia y difícil, ya que hay que contar con los conocimientos necesarios para ejecutarla correcta y coherentemente.

\section{Cualidades y el contexto en el ambiente virtual}

La observación del fenómeno informacional en el contexto de la educación superior, pero en diferentes ambientes que son el impreso y el virtual, permite identificar que cada caso requiere de una configuración de procesos y cualidades específicas para desenvolverse eficientemente en cada campo. Por ejemplo, en el ambiente impreso se visualizó que la búsqueda de la información es importante, pero no compleja, caso contrario al ambiente virtual, donde este proceso se torna fácil a primera vista, pero en realidad es un camino difícil y lleno de obstáculos. Y esta misma demanda cognitiva e instrumental para la búsqueda de la información digital se liga con dos cualidades predominantes en el contexto de la educación superior: la ética y la ciudadanía.

La ética se coloca como esencial en un ambiente donde los estudiantes, aparte de lidiar con las "burbujas de ocio" y/o la desconcentración informacional, también deben concientizarse sobre el uso de la información. De acuerdo a Pineda, Hennig, Segovia, Díaz, Sánchez, Otero, y Paul (2012), una vez que el alumno ha concretado la localización y selección de la información a través de la lectura analítica y crítica, viene el reto de manipularla con las necesarias normas de derechos de autor para lograr el uso apropiado de ella. Efectivamente, la facilidad con la que se puede acceder y utilizar la información que existe en Internet, invita negativamente a los actos de plagio si es que el estudiante no tiene una formación en estos temas, puesto que en la mayoría de los casos estos llegan a la educación superior sin conocer cómo citar o referenciar los datos que encontraron en la red y con los que se están apoyando para realizar sus trabajos escolares.

Asimismo, en un ambiente donde los dispositivos digitales portátiles son cada vez más accesibles a las personas, y cada vez más intitutivos para usarse, se convierten en herramientas de doble filo, puesto que las personas pueden efectuar un uso positivo o contraproducente para la sociedad en general. Por esta razón, la ética se posiciona como emergente para la conformación de una ciudadanía que efectúe prácticas que verdaderamente beneficien a la comunidad, y no las afecte (Orozco y García, 2017). Es lo que Fueyo, Rodríguez y Hoeschsman (2018) señalan como la construcción de una globalidad ciudadana que sepa cómo aprovechar las TIC que tiene a su alrededor para crecer personal y socialmente, esto es, ejercer una dinámica real que conlleve a la anhelada sociedad de la información y del conocimiento.

\section{Conclusiones}

Se han puesto sobre la mesa las piezas que configuran a la "alfabetización", concepto que se define - a partir de lo expuesto y analizadocomo aquél conjunto de conocimientos, habilidades, actitudes, prácticas, y especialmente procesos y cualidades, que convergen para que una persona sepa cómo codificar, asimilar, utilizar y reproducir la información que necesite de ciertos contextos y ambientes para desempeñarse, lo más productiva y eficientemente posible, siempre a favor del desarrollo personal y el crecimiento social. Asimismo, se debe asimilar que, si bien la "alfabetización" involucra de facto los procesos de lectura y escritura, estos no deben encerrarse en la mera codificación de la letra escrita. Hay mucha más información alrededor de los objetos que pueden leerse y escribirse - o lo que puede ser equivalente a consumirse y producirse-, de esta forma se rompe con los límites de la alfabetización para colocarla como un fenómeno que se apoya del fenómeno informacional, pero que no puede igualarse conceptualmente con él.

Por lo tanto, si se analizan desde esta perspectiva a ambos conceptos (la alfabetización y lo informacional) se logra identificar que trabajar la propuesta de una "alfabetización informacional" es caer en un error ontológico, por dos razones: en primer lugar, porque la sola expresión implica caer en una especie de redundancia, y en segundo lugar, porque se presenta a lo "informacional" como lo nuevo cuando en realidad es una parte de la "alfabetización". Esto se puede hacer más gráfico retomando el ejemplo de la agronomía e imaginando que algún autor propone la definición de una "agricultura agrónoma", hecho que con solo leerlo detona una incoherencia conceptual. 
Esta misma percepción automática debería pasar al momento de leer el compuesto "alfabetización informacional", pero se ha desviado tanto el entendimiento del término "alfabetización" que cada noción con este concepto, de inicio, se valida como correcto y pertinente.

Así surgen un sin fin de "alfabetizaciones": alfabetización automotriz, alfabetización fotográfica, alfabetización artística, alfabetización industrial, etc., "conceptos" que en lugar de aclarar el fenómeno de la "alfabetización", solo lo han abrumado. Y es que hay que resaltar la idea de una "alfabetización" como concepto único que no puede ni debe re conceptualizarse, sino más bien, que puede estar presente en cualquier contexto, ambiente y práctica social, por lo que pensar en una "alfabetización" en o para, es más compatible con el entusiasmo de concebir siempre a la "alfabetización" como única. Por ejemplo, habría una alfabetización en lo o para lo musical, y no una "alfabetización musical", de esta manera se estaría ejerciendo una dinámica de estudio más ordenada en el fenómeno de la "alfabetización". En este sentido, al entender que la "alfabetización" es única, que es configurable pero no re conceptualizable, se llega a poner en duda el pensamiento que expone la existencia de lo que se define actualmente como "multialfabetización". A pesar de que autores como Posadas (2010) y García (2015) mencionan que una persona puede hacer uso de dos alfabetizaciones para llevar a cabo una tarea específica, encontramos que tal afirmación no tiene concordancia con la visión de la "alfabetización" como concepto único.

Esta misma incomprensión y falta de análisis en el concepto ha originado un efecto dominó con propuestas no coherentes ontológicamente como el mencionado compuesto "multialfabetización". Cabe aclarar que no se están descalificando los esfuerzos en el estudio de este fenómeno, sino que se señala la idea de una "alfabetización" como concepto único y configurable a partir de lo que se desea codificar y realizar. Porque todo es información, por lo que todo puede leerse y escribirse o consumirse y producirse.

Por último, se aclara que el discurso de este artículo, versa sobre una propuesta conceptual, la cual está dirigida hacia una mejor comprensión del tema de alfabetización; en este sentido, se colca como un aporte relevante al conocimiento, puesto que como ya se ha mencionado, plantear una delimitación conceptual funge como un elemento importante para la apertura de un camino más claro y fluido en el estudio de la alfabetización, ya que esta debería ser la dinámica investigativa y de divulgación del conocimiento, la de aportar para comprender, y no, exponer y confundir, lo cual puede asemejarse a contar con un camino que debe mantenerse limpio de maleza u otros obstáculos que dificulten el tránsito hacia delante.

\section{Agradecimientos}

Este artículo se desprende de la investigación titulada Literacidad Visual Digital en Estudiantes Universitarios que actualmente se realiza en el Doctorado en Investigación e Innovación Educativa (DIIE) de la Benemérita Universidad Autónoma de Puebla, bajo la asesoría del Dr. Abraham Moctezuma Franco. Se debe resaltar que este documento agradece y da crédito de manera pública al apoyo institucional y académico recibido para la ejecución de este proyecto.

\section{Referencias bibliográficas}

Aguaded, I. y Romero, L. (2015). Mediamorfosis y desinformaciónenlainfoesfera:Alfabetización mediática, digital e informacional ante los cambios de hábitos de consumo informativo. Teoría de La Educación. Educación y Cultura En La Sociedad de La Información, 16(1), 44-57. Recuperado de https://www.proxydgb. buap.mx:2168/10.14201/eks20151614457

Bawden, D. (2002). Revisión de los conceptos de alfabetización informacional y alfabetización digital. Revista Anales de Documentación, (5), 361-408. Recuperado de: http://revistas. um.es/analesdoc/article/view/2261

Braslavsky, B. (2003). Lectura y Vida. Revista latinoamericana de lectura. 2-17. Recuperado de www.lecturayvida.fahce.unlp.edu.ar/ numeros/a24n2/24 02 Braslavsky.pdf

Carlino, P. (2013). Alfabetización Académica Diez Años Después. Revista Mexicana de Investigación Educativa, 18(57), 355-381. Recuperado de https://www.proxydgb.buap. mx:2057/login.aspx?direct $=$ true $\& \mathrm{db}=\mathrm{ehh} \& \mathrm{~A}$ $\mathrm{N}=91024925 \&$ lang=es\&site=ehost-live

Carlino, P.(2017). Dos variantes de laalfabetización académica cuando se entrelazan la lectura y la escritura en las materias. Signo y Pensamiento, 36(71), 16-32. Recuperado de https://www. proxydgb.buap.mx:2168/10.11144/Javeriana. syp36-71.dvaa 
Carrasco, A. y López, G. (2014). Lenguaje y Educación. Temas de investigación educativa en México. D.F. México: Fundación SM

Cassany, D. (2006). Tras las líneas. Sobre la lectura contemporánea. Barcelona, España: Editorial Anagrama, S. A.

Del Pilar, M. y Pirela, J. (2016). La alfabetización como estrategia de inclusión socio-digital: entre diversidades y mutaciones conceptuales. Enl@ce: Revista Venezolana de Información, Tecnología y Conocimiento, 13(3), 93-113. Recuperado de https:/www.proxydgb.buap. mx:2057/login.aspx?direct=true $\& \mathrm{db}=$ fua $\& A$ $\mathrm{N}=121730831 \&$ lang=es\&site $=$ ehost-live

Fernández, J. y Piña, L. (2014). El Oficio Del Escritor Académico. Revista Mexicana de Investigación Educativa, 19(60), 187-212. Recuperado de https://www.proxydgb.buap. mx:2057/login.aspx? direct $=$ true $\& d b=a 9 h \& A$ $\mathrm{N}=94924673$ \&lang=es\&site=ehost-live

Fernández, J., y Piña, L. (2014). El Oficio Del Escritor Académico. Revista Mexicana de Investigación Educativa, 19(60), 187-212. Recuperado de https://www.proxydgb.buap. mx:2057/login.aspx?direct $=$ true $\& d b=a 9 h \& A$ $\mathrm{N}=94924673$ \&lang=es\&site=ehost-live

Ferreiro, E. (1997). Alfabetización. teoría y Práctica. México: Siglo XXI Editores.

Fueyo,A., Rodríguez, C. y Hoeschsman, M. (2018). Construyendo Ciudadanía Global en Tiempos de Neoliberalismo: Confluencias entre la Educación Mediática y la Alfabetización Digital. Revista Interuniversitaria de Formación Del Profesorado, 32(1), 57-68. Recuperado de https://www.proxydgb.buap. mx:2057/login.aspx?direct=true $\& d b=$ fua $\& A$ $\mathrm{N}=129381440$ \&lang=es\&site $=$ ehost-live

García, H. (2015). Multialfabetización en la sociedad del conocimiento: competencias informacionales en el sistema educativo. Revista Lasallista de Investigación, 12(2), 225-241. Recuperado de https://www. proxydgb.buap.mx:2057/login.aspx?direct $=$ tr ue\&db=a9h\&AN=119193300\&lang=es\&site $=$ ehost-live

Gómez, A. y Gómez, M. (2015). Escritura ortográfica y mensajes de texto en estudiantes universitarios. Perfiles Educativos. 38(150), 91-104. Recuperado de http://www.iisue. unam.mx/perfiles/articulos/2015/n150a2015/ mx.peredu.2015.n150.p91-104.pdf

Hernández, D., Cassany, D. y López, R. (Coordinadores) (2018). Prácticas de lectura y escritura en la era digital. Córdoba, Brujas,
México: Asociación Civil Social TIC 2018. (Háblame de TIC - Ramírez, Alberto; Casillas Alvarado, Miguel Angel; Vol. 5)

Lau, J. y Cortés, J. (2009). Habilidades informativas: convergencia entre ciencias de información y comunicación. Revista Científica de Educomunicación, 32 (16). pp. 21-30.

López, F.y Parker, C.(2009).Alfabetismocientífico, misión de la universidad y ciudadanía: ideas para su construcción en los países en vías de desarrollo. Avaliação: Revista da Avaliação da Educação Superior (Campinas), 14(2), 267-290. Recuperado de https://dx.doi. org/10.1590/S1414-40772009000200003

López, G. y Pérez, C. (2014). Debates actuales en torno a los conceptos de alfabetización, lectura escruta y literacidad. En A. Carrasco, y G. López (Ed.), Lenguaje y educación. Temas de investigación educativa en México (pp. 21-45). D.F., México: Fundación SM

Martín, M. (2018). Alfabetización informacional y biblioteca neoliberal: Hacia un nuevo paradigma. Informacion, Cultura y Sociedad, (39), 129-138. Recuperado de https://www. proxydgb.buap.mx:2057/login.aspx?direct=tr ue\& $\mathrm{db}=\mathrm{a} 9 \mathrm{~h} \& \mathrm{AN}=133784837$ \&lang=es\&site $=$ ehost-live

Molina, M. y Valdés, M. (2010). Alfabetización informacional, innovación evaluación como funciones de la biblioteca universitaria del siglo XXI: visión desde un enfoque cualitativo. Ibersid, 81-91. Recuperado de https://www.proxydgb.buap.mx:2057/login. aspx? direct $=$ true $\& \mathrm{db}=\mathrm{a} 9 \mathrm{~h} \& \mathrm{AN}=54321701 \&$ lang $=$ es \& site $=$ ehost-live

Orozco, A. y García, M. (2017). Desarrollo De Habilidades Cognitivas Para LaAlfabetización Digital. Revista de La Alta Tecnología y Sociedad, 9(4), 138-145. Recuperado de https://www.proxydgb.buap.mx:2057/login. aspx?direct $=$ true $\& \mathrm{db}=\mathrm{zbh} \& \mathrm{AN}=123819734$ \&lang $=$ es \&site $=$ ehost-live

Pineda, C., Hennig, C., Segovia, Y., Díaz, D., Sánchez, M., Otero, M. y Paul, G. (2012). Alfabetización Informacional en La Educación Superior Virtual: Logros Y Desafíos. Información, Cultura y Sociedad, (26), 83-104. Recuperado de https://www. proxydgb.buap.mx:2057/login.aspx?direct=tr ue $\& \mathrm{db}=\mathrm{a} 9$ h\&AN $=78577425 \&$ lang $=$ es \& site $=$ ehost-live

Pini, M. (2019). Políticas de alfabetización digital. Educación e inclusión. (Spanish). Cuadernos 
Del Centro de Estudios de Diseño y Comunicación, 19(72), 95-107. Recuperado de https://www.proxydgb.buap.mx:2057/ $\underline{\text { login } . a s p x ? \text { direct }=\text { true } \& \mathrm{db}=\text { asu } \& A N=13132}$ 4723\&lang=es\&site $=$ ehost-live

Posadas, C. (2010). Multialfabetización y redes sociales en la universidad. RUSC: Revista de Universidad y Sociedad Del Conocimiento, 7(2), 17-27. Recuperado de https://www. proxydgb.buap.mx:2057/login.aspx?direct $=$ tr ue\&db=ehh\&AN=89006577\&lang=es\&site= ehost-live

Reis, C., Pessoa, T., y Gallego, M. (2019). Alfabetización y competencia digital en Educación Superior: Una revisión sistemática. Revista de Docencia Universitaria, 17(1), 45-58. Recuperado de https://www.proxydgb. buap.mx:2168/10.4995/redu.2019.11274

Sánchez, A., Sánchez, L., Méndez, J. y Puerta, C. (2013). Alfabetización académico- investigativa: citar, argumentar y leer en la red. Revista Lasallista de Investigación, 10(2), 151-163. Recuperado de https://www. proxydgb.buap.mx:2057/login.aspx?direct=tr $\underline{u e} \& \mathrm{db}=\mathrm{a} 9 \mathrm{~h} \& \mathrm{AN}=94266737 \&$ lang $=$ es\&site= ehost-live

Scalerandi, M. (2018). Lectura crítica en entornos digitales en el nivel superior: ¿nueva práctica letrada con características propias?. Revista Cientifica de la Universidad de Belgrano. 1(2), 109-119.

Vega, G. y Quijano, Á. (2010). Comunidades de práctica y alfabetización informacional. Ibersid, 93-103. Recuperado de https://www. proxydgb.buap.mx:2057/login.aspx?direct=tr

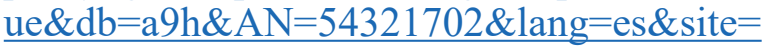
ehost-live 